\title{
Visualizing Design Exclusion Predicted by Disability Data: A Mobile Phone Case Study
}

\author{
Sam Waller, Pat Langdon and John Clarkson \\ University of Cambridge, Engineering Design Centre, Department of Engineering \\ Trumpington Street, Cambridge, CB2 1PZ \\ \{sdw32,pml24,pjc10\}@cam.ac.uk
}

\begin{abstract}
Disability data can help to predict the number of people that will be unable to use a particular product. The greatest benefits of this prediction are the design insights that help to reduce exclusion and thereby improve the product experience for a broad range of people. This paper uses a mobile phone case study to demonstrate how a set of visualization outputs from an exclusion audit can generate prioritized design insights to reduce exclusion, particularly when multiple tasks place demands on multiple capabilities.
\end{abstract}

Keywords: Inclusive design, calculating exclusion

\section{Introduction}

The Cambridge Engineering Design Centre is unique in developing analytical tools that can quantitatively assess the inclusive merit of different design decisions or products, according to the number of potential users that would be excluded: such tools have the potential to greatly assist the implementation of inclusive design in businesses [5]. In addition to those excluded from using a product, many more people will experience difficulty or frustration, so reducing the number of people excluded can improve the experience for a wide range of users [8].

Performing tasks with products typically involves a perception-cognition-action cycle, where the user perceives and interprets information from the product, that further thinking processes use, together with reference to long-term memory, to choose an intended action. All of these processes are affected by the context of use, and the user's ability to successfully perform this cycle impacts on whether they will find the product easy, difficult or impossible to use [11]. The tools that comprise an exclusion audit therefore intend to present population data about users' context dependent abilities to perceive, think and act, so that designers choosing the interface attributes of mainstream products and services can make better informed decisions to reduce the number of people who find the usage difficult or impossible [7].

Exclusion auditing is intended to complement other tools for evaluating inclusive merit, such as expert opinion [9], user trials [1] and impairment simulators [10]. In combination, these tools provide a holistic approach to discover the causes of design 
exclusion, and identify appropriate design improvements. An ideal data source for evaluating design exclusion would:

- Be representative of a national population, and be customizable towards specific markets of interest.

- Contain data that covers users' abilities to perceive, think and act in real-world contexts.

- Be simple to apply and directly relevant to product interaction.

- Have sufficient scope to incorporate the variation of capability typically found within developed societies, yet sufficient granularity to predict the difference in exclusion between alternative mainstream products.

Surveys that are nationally representative are usually carried out to collect data about one specific aspect of perceiving, thinking or acting. However, product usage engages these abilities in combination, and it is not possible to combine several separate surveys together, so data that is suitable for estimating design exclusion is hard to find. Indeed no UK dataset exists that satisfies all of the criteria required to evaluate design exclusion [2]. The latest $i \sim$ design research program [6] aims to address this shortcoming directly, by designing and undertaking a national survey specifically for this purpose. Until the results from this survey become available, the Office of National Statistics 1996/97 Disability Follow-up Survey (DFS) [4] remains the best available data source for estimating UK design exclusion [11].

This paper first examines the DFS in more detail, and then presents several ways of visualizing the results when this survey is used to predict design exclusion for one or more tasks. The objectives are to understand how many people are excluded, why those people are excluded, and to provide design insights for how they could be included. The exclusion audits presented here-in are based on the DFS, but the techniques are intended to apply to alternative data sources that could predict design exclusion, perhaps for different countries or for future data collected.

\section{The Disability Follow-up Survey}

The survey was conducted in 1996/97 in order to plan welfare support for people with disabilities. The survey contained questions that were grouped together in 13 ability categories, 7 of which are most relevant for product interaction, namely seeing, hearing, intellectual function, communication, locomotion, reach \& stretch, and dexterity (for the sake of clarity, seeing and intellectual function will be referred to in this paper as vision and thinking). Within each category, the questions were intended to cover the full range of severity of quality of life impairment.

For example, the dexterity questions range from disabilities with mild effect on quality of life, such as "can you tie a bow in laces or string without difficulty?" to disabilities with severe effect on quality of life, such as "can you pick up and hold a mug of coffee with either hand?". The complete set of ability levels can be found online [3].

The collection of self-reported ability levels for each of the 7200 survey participants in each of the seven categories forms the DFS capability database, the structure of which is illustrated in Table 1. Each person surveyed has a unique 
Table 1. Extract from the ONS dataset, where the 4th row represents a person with full ability, according to the definition used in the survey

\begin{tabular}{ccccccccccc}
\hline ID & $\begin{array}{l}\text { Multi- } \\
\text { plier }\end{array}$ & Age & Sex & Vision & $\begin{array}{l}\text { Hear- } \\
\text { ing }\end{array}$ & $\begin{array}{l}\text { Think- } \\
\text { ing }\end{array}$ & $\begin{array}{l}\text { Comm- } \\
\text { unication }\end{array}$ & $\begin{array}{l}\text { Loco- } \\
\text { motion }\end{array}$ & $\begin{array}{l}\text { Reach \& } \\
\text { stretch }\end{array}$ & $\begin{array}{l}\text { Dex- } \\
\text { terity }\end{array}$ \\
\hline 1 & 1894 & 24 & M & V9 & H8 & T9 & C6 & L14 & R11 & D9 \\
2 & 1312 & 61 & M & V10 & H6 & T12 & C6 & L9 & R11 & D12 \\
3 & 1865 & 49 & F & V10 & H9 & T12 & C6 & L8 & R7 & D12 \\
4 & 1267 & 35 & F & V10 & H9 & T12 & C6 & L14 & R11 & D12 \\
\hline
\end{tabular}

identifier, and statistical measures were used to calculate the number of people in the country represented by that person (labeled multiplier).

\section{Estimating Exclusion from the DFS}

The number of people excluded from using a product or service will depend on the demands that it makes on their capabilities, which depends on the goal the user wants to achieve with the product, and the tasks needed to perform that goal. The tasks that are actually required, and the level of ability required to perform those tasks will depend on the context of use, which includes both the current environment and the current state of all items involved in achieving the goal. An exclusion analysis is typically based on a single set of assumptions for the context of use, and the results would change for a different set of assumptions.

This paper focuses on visualizing the exclusion results for various different tasks that are associated with using a product. The visualizations are intended to help practical decision makers generate prioritized design insights to reduce exclusion. However, even with a single task, understanding the breakdown of design exclusion will likely require a numerate background, and the complexity of the breakdown rises exponentially with the number of tasks assessed, so specific training may be needed to understand how to best interpret the results when multiple tasks are involved.

To estimate the exclusion associated with a task, the demands placed on each DFS capability must first be assessed in a manner that is directly compatible with the DFS database, then the demand assessment can be used to evaluate exclusion. This assessment procedure is described in detail elsewhere [11], and summarized here.

The assessor must first break down the task in question and examine the demands it places on each of the seven DFS ability categories. For each category in turn, the assessor should examine all the statements in all the ability levels of that category, and judge whether that capability is required to perform the task. A demand of zero indicates that capability is not required.

If some level of that capability is required, the assessor should then consider the lowest ability level within the capability of interest, and judge whether a person with that ability level would be able to perform the task. In most cases, a person with such low ability would be unable to perform the task, in which case the judgment should be 
repeated for each higher ability level, until the threshold is identified where the task first becomes possible. The demand on that capability is now defined as the ability level that is one lower than the threshold just identified.

This procedure is repeated to identify the demand on each of the seven DFS ability categories. For example, charging a typical mobile phone may result in the demands [V9;H0;T1;C0;L8;R5;D6], where each letter represents one of the seven DFS ability categories, each number indicates how much demand is placed on that category, higher numbers refer to products that are more difficult to use, and the meaning of each demand is explained in more detail later on. The DFS capability database can then be used to predict the proportion of the UK population who would be excluded from the task, taking due account of the people who may be excluded because the demands are too high in several ability categories.

\section{Visualizing the Excluded Population for One Task}

The task of charging a typical mobile phone is now examined in more detail, assuming that the user is holding the phone in his or her hand, and the charger jack is lying on the floor. Recall that the demand summary for charging a typical mobile phone is $[\mathrm{V} 9 ; \mathrm{H} 0 ; \mathrm{T} 1 ; \mathrm{C} 0 ; \mathrm{L} 8 ; \mathrm{R} 5 ; \mathrm{D} 6]$, where the vision demand reflects identifying a small, black, poorly labeled charger socket from the rest of the chassis of the phone, the thinking demand reflects performing a simple task, the locomotion demand reflects bending down to pick up the charger jack, the reach \& stretch demand reflects briefly holding both hands out a short distance from the body, and the dexterity demand reflects holding the phone in one hand, then aligning and inserting the charger jack with the other.

The DFS capability database predicts that 5.0 million UK adults will be excluded from attaching the charger to this typical phone. Of the excluded adults, some of them are excluded because only one demand exceeded their capabilities; others are excluded because several demands exceeded their capabilities, shown in Fig. 1(a). In order to help understand which demands are causing the most difficulty for the most people, Figs 1(b) and 1(c) show exclusion predictions for two alternative scenarios. Firstly, supposing it was possible to redesign the task to eliminate the demand on one particular capability, Fig. 1(b) indicates how much the overall exclusion would reduce as a result. Secondly, supposing the task was redesigned to eliminate the demand on every capability except one, Fig. 1(c) shows how many people would be excluded by the one remaining demand.

In general, Fig. 1(b) represents a more realistic scenario; however its usefulness depends on how many of the excluded adults were unable to achieve the task because of a demand on just one capability. This number inevitably drops when more capabilities are involved, in which case the bars presented in Fig. 1(b) would tend to zero length, and Fig. 1(c) becomes the more useful predictor of which capability demand(s) are causing the most difficulty for the most people. 
(a) Breakdown of the total population

Excluded because of a demand on just one capability

Excluded because of the demands on several capabilities

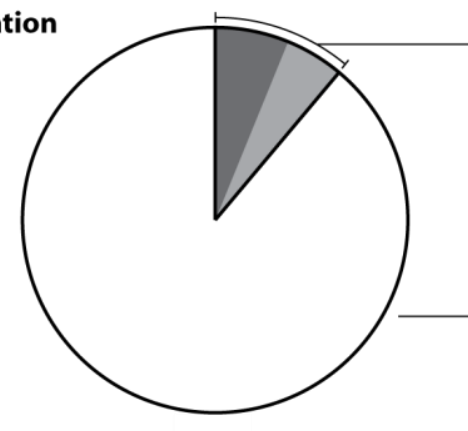
5.0 million $\mathrm{GB}$ adults (11\%) are excluded by the task

40.6 million GB adults (89\%) are able to achieve the task

(b) If the task could be redesigned to eliminate the demand on just one capability, by how much would the overall exclusion reduce?

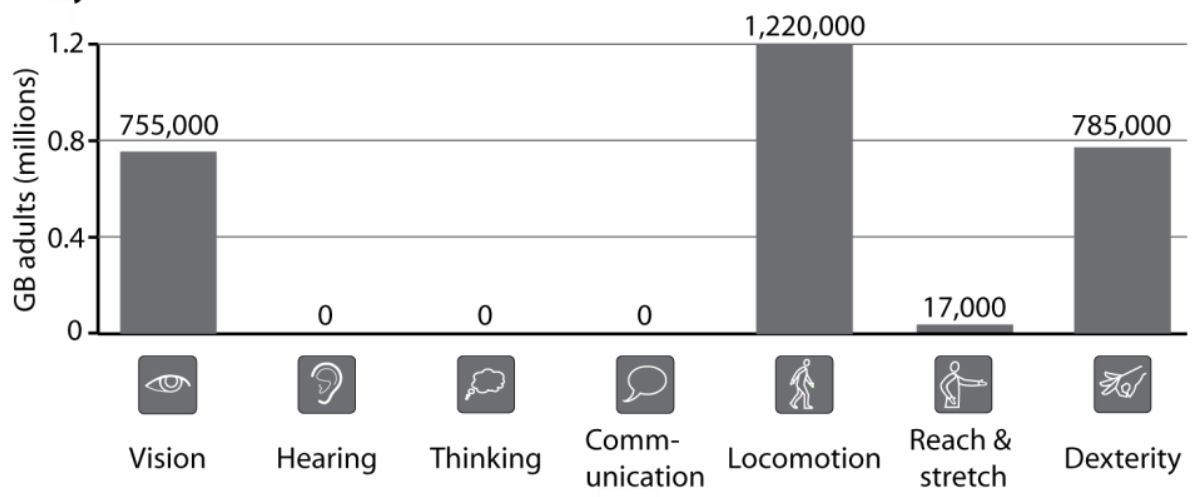

(c) If the task could be redesigned to eliminate the demand on every capability except one, how many people would be excluded because of the one remaining demand?

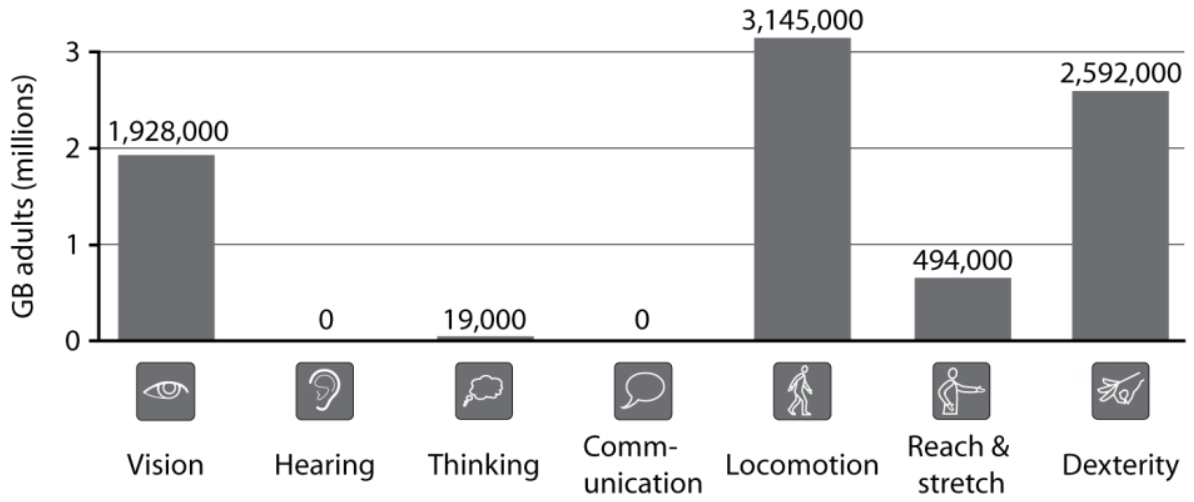

Fig. 1. Visualising the results from an exclusion analysis for charging a typical mobile phone. (a) shows the total number excluded, while (b) and (c) show exclusion figures for two alternative scenarios. Additionally, (b) is equivalent to a further breakdown of the people in (a) who were excluded because of a demand on just one capability. 
In this particular case, Fig. 1(a) shows that of the excluded adults, about half of them were excluded because of a demand on just one capability, and Figs 1(b) and (c) both suggest that reducing the locomotion demand has the greatest potential to reduce the number of people who are unable to attach the charger. The locomotion demand occurs because the user typically has to bend down to pick up the charger jack, so one way to achieve this reduction might be to supply the phone with a freestanding desk charger, or ensure that such an accessory is readily available with a mainstream price point.

\section{Visualizing the Excluded Population for Multiple Tasks}

Using a product will typically involve many tasks, and the previous example for charging a mobile phone is now extended to consider inserting the SIM card, turning the phone on, and receiving a call. For all of these tasks it is assumed that the user is sitting at a desk, with typical indoor lighting and no background noise. These tasks are now examined in further detail, and the demand summaries for each task are shown graphically in Fig. 2.

For inserting the SIM card, it is additionally assumed that the user is familiar with the general concept of inserting a SIM card, but will use the instruction book to guide them through the task for this particular phone. The demand summary is therefore [V9;H8;T5;C0;L0;R5;D11], where the vision demand reflects reading the instruction manual, and identifying the small parts of the hatch that encloses the SIM card,
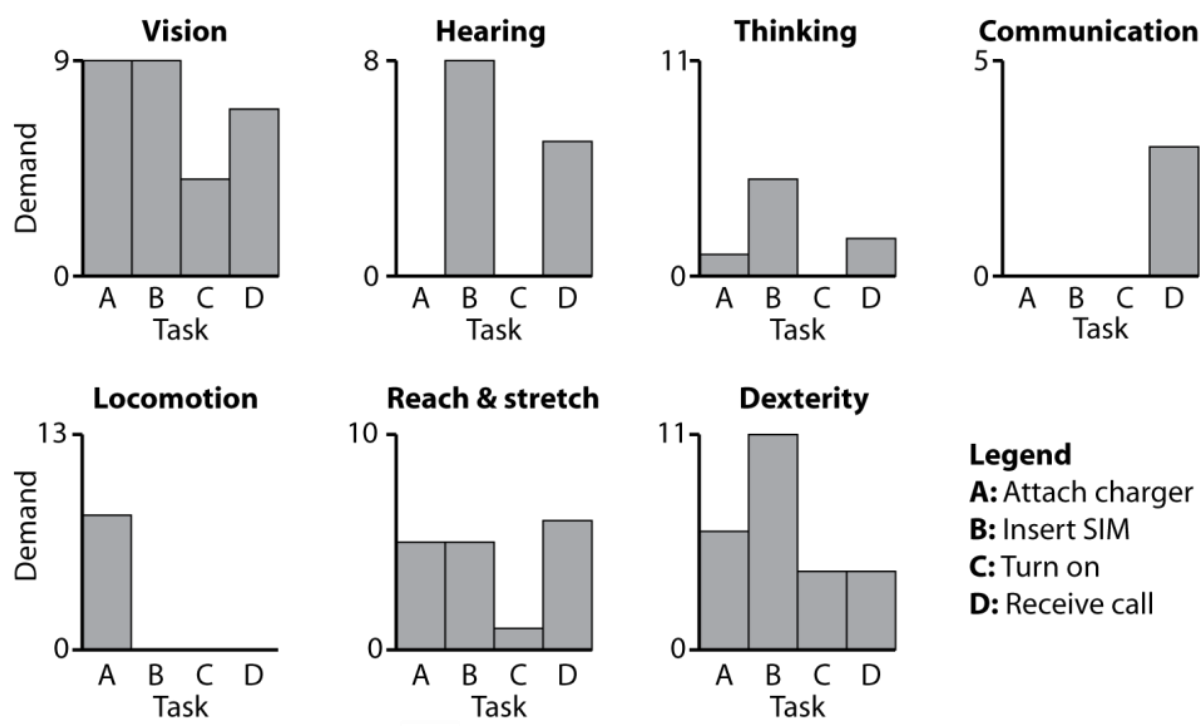

Fig. 2. The demands made by each task on each capability. Although the different scales have different numbers of levels within them, each graph has been drawn with the same vertical height, to reflect the original survey's intended equivalence for the full range of each scale. 
the hearing demand reflects hearing the hatch click back into place (this confirmation is difficult to achieve by vision or touch), the thinking demand reflects reading short passages of text, then planning and executing a structured series of activities, the reach \& stretch demand is the same as for attaching the charger, but the dexterity demand is higher because of the need to manipulate and align small parts with both

(a) Breakdown of the total population

Excluded because the demands of just one task exceeded their ability

Excluded because the demands on several tasks exceeded their ability

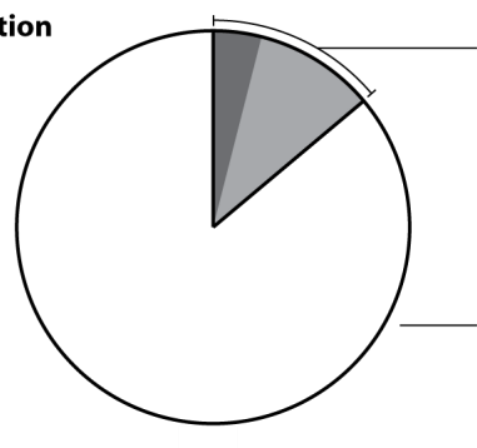

6.3 million $G B$ adults (14\%) are unable to achieve all 4 tasks

39.3 million $\mathrm{GB}$ adults $(86 \%)$ are able to achieve all 4 tasks

(b) If the product could be redesigned so that the user is no longer required to perform one of the tasks, by how much would the overall exclusion reduce?

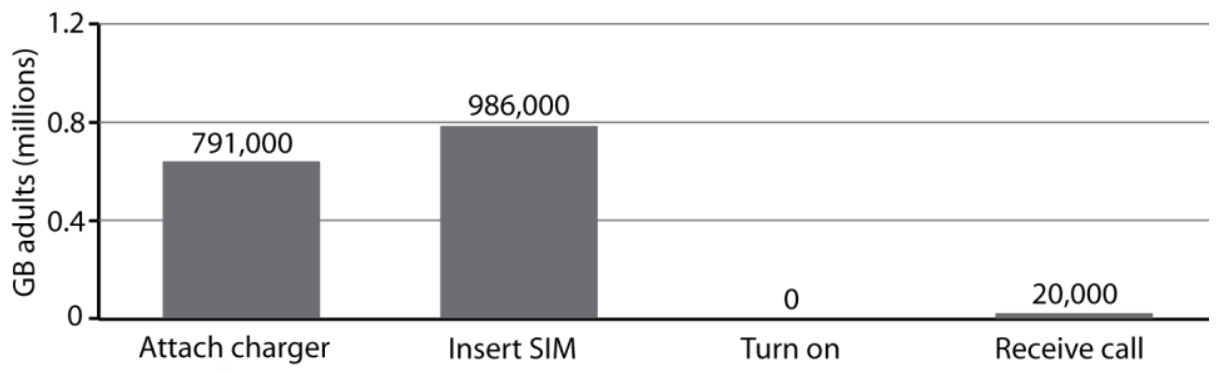

(c) If each task is considered separately, how many people are excluded?

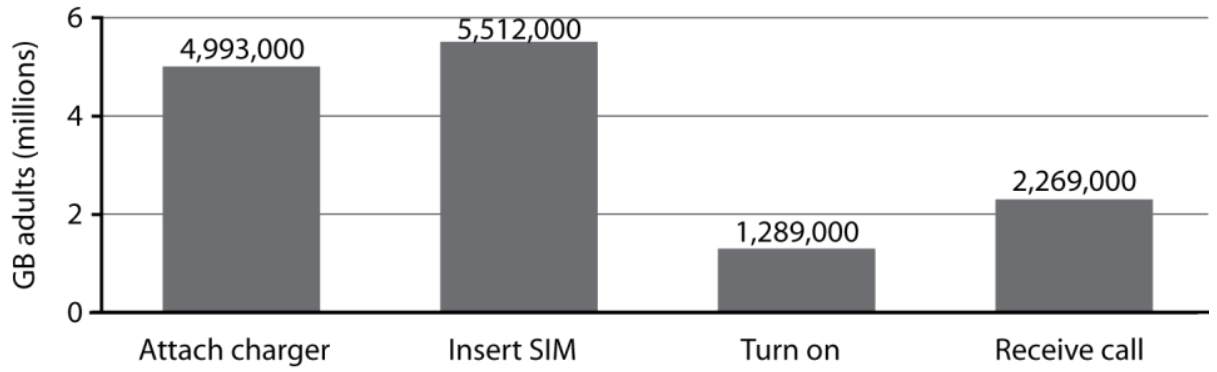

Fig. 3. Visualizing the results from an exclusion analysis for four different tasks with a typical mobile phone. (a) shows the total number excluded, while (b) and (c) show exclusion figures for two alternative scenarios. Additionally, (b) is equivalent to a further breakdown of the people in (a) who were excluded because of the demands of just one of the tasks. 
hands.For turning the phone on the demand summary is [V4;H0;T0;C0;L0;R1;D4], where the vision demand reflects identifying a silver power button on the top of the phone, the reach \& stretch demand reflects briefly holding one hand out a short distance from the body, and the dexterity demand reflects pushing the power button.

For answering a call, it is additionally assumed that the phone's loudspeaker is enabled, so the phone does not have to be held up to the head. The demand summary is therefore $[\mathrm{V} 7 ; \mathrm{H} 5 ; \mathrm{T} 2 ; \mathrm{C} 3 ; \mathrm{L} 0 ; \mathrm{R} 1 ; \mathrm{D} 4]$, where the vision demand reflects identifying the button to answer the call, the hearing demand reflects hearing speech from the phone's earpiece, the thinking and communication demands reflect maintaining a conversation, the reach \& stretch demand reflects holding one hand out a short distance from the body, and the dexterity demand reflects pushing the button to answer the call.

Assuming that performing any of the tasks doesn't make any of the others more difficult (i.e. no fatigue effects), the DFS capability database predicts that 6.3 million adults will be unable to perform the set of four tasks. Of the excluded adults, some are excluded because the demands of just one task exceeded their ability; others are excluded because the demands of several tasks exceeded the ability, shown in Fig. 3(a). Similar to the visualizations presented in Fig. 1, Figs 3(b) and (c) show the results from two alternative scenarios that help to understand which task(s) are causing the most difficulty for the most people. The usefulness of Fig. 3(b) also similarly decreases as the number of adults who were excluded because of just one task decreases, which will inevitably occur as the number of tasks increases.

In order to fully understand the reasons leading to exclusion it may be useful to split the people unable to perform the set of tasks into 15 different categories, according to whether they are able or unable to achieve all the different combinations of the 4 tasks being considered, as shown in Fig. 4.

In this particular case, Fig. 3(a) indicates that of the excluded adults, only a small proportion of them were excluded because of just one of the tasks, which limits the insights available from Fig. 3(b), and also means that reducing the demands of just

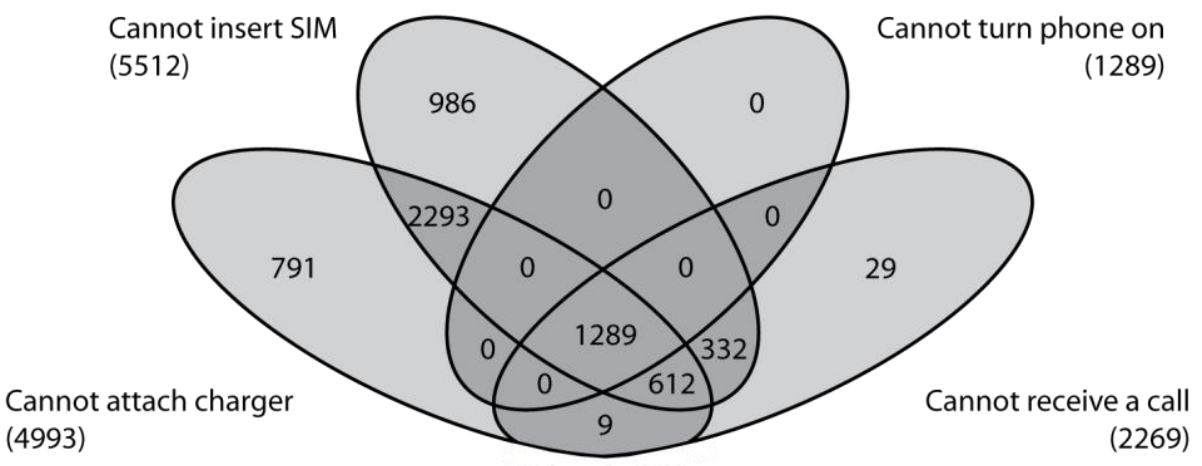

Fig. 4. Breakdown of the 6.3 million adults who are unable to perform four tasks with a typical mobile phone. All values are '000s GB adults, and numbers may not sum exactly due to rounding. The numbers within the areas that "stick out" from all the others are also shown in Fig. 3(b), while the number beside each seperate task is also shown in Fig. 3(c). 
one task on its own will not make much difference to the overall exclusion. Figure 3(c) shows that considered independently, the tasks of attaching the charger and inserting the SIM are the most significant causes of exclusion, however the best insight comes from Fig. 4, which shows that of 15 different possible combinations, a clear majority (2.3 million adults) can turn the phone on and can make a call, but cannot insert the SIM and cannot attach the charger. Reducing the demands of both attaching the charger and inserting the SIM is therefore the most effective way to reduce the overall exclusion.

To further prioritize which aspects of attaching the charger and inserting the SIM card require the greatest attention, it may also be helpful to take each separate demand associated with each separate task in turn, and consider how many adults would be excluded if every other demand was eliminated, shown in Fig. 5. From Fig. 3, and Fig. 5, attaching the charger is the only task that requires locomotion ability, and inserting the SIM card requires more hearing and dexterity ability than any other task, so reducing these particular demands could be the most effective way to reduce the overall design exclusion. However, further iterative analyses would typically be recommended to refine the initial assumptions and check the true benefit of any proposed design solutions that would reduce these demands.

Additionally, comparing the relative magnitudes of the demands in Fig. 2 and the numbers of people excluded in Fig. 5 indicates the relative prevalence of capability loss within each category, according to the intended equivalence of each scale within the underlying survey. For this particular example the DFS considers the demand of bending down as roughly equivalent to communicating with a friend, yet the corresponding exclusion is nearly 10 times higher for bending down.
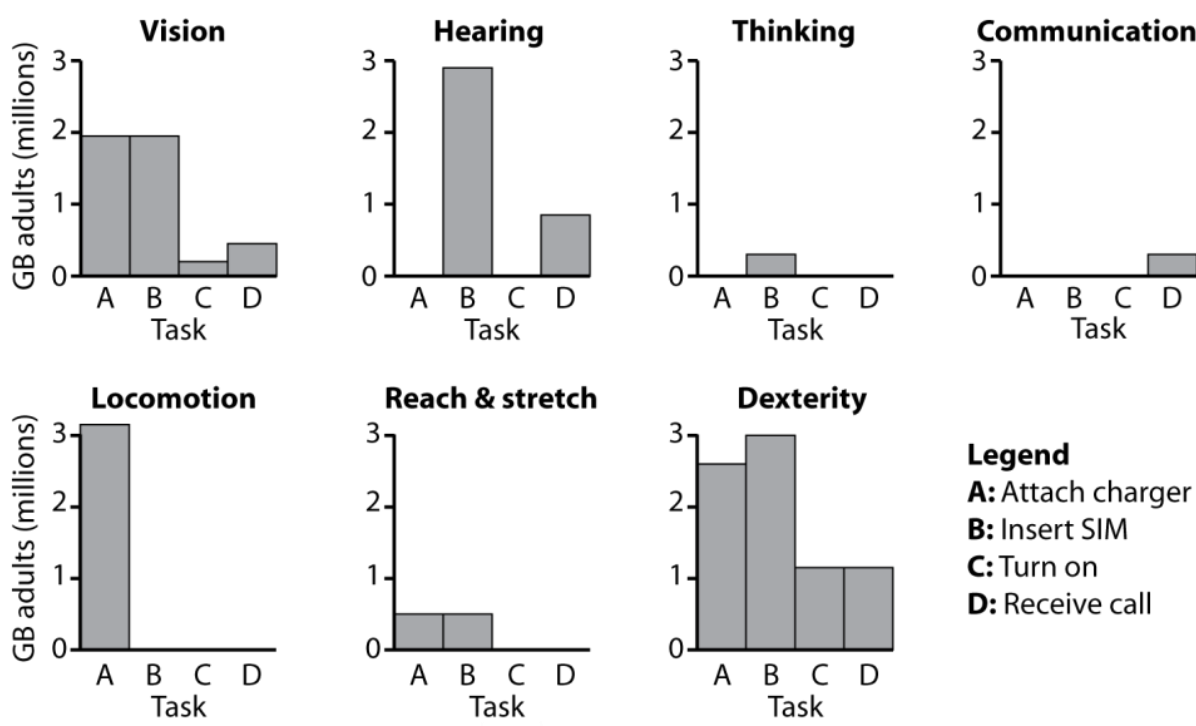

\section{Legend}

A: Attach charger

B: Insert SIM

C: Turn on

D: Receive call

Fig. 5. For each separate demand associated each task, these graphs show how many people would be excluded if every other demand was eliminated. For the task of attaching the charger, the data presented here is identical to that shown in Fig. 1(c). 


\section{Conclusions and Further Work}

An exclusion audit uses an assessor's judgment to compare a particular task against generic capability data, and the authors are currently planning research to investigate the reliability and validity of such judgments, and investigate how training an assessor with different tools and techniques could support this process. Although the best available accuracy in estimating exclusion from the DFS data is limited, the relative magnitudes of the different causes of exclusion and the design solutions they inspire are expected to retain some validity.

The hypothetical example presented here showed how visualizing the breakdown of exclusion can lead to real and prioritized design insights. Considering design changes in terms of the number of potential customers who might benefit provides a compelling business case for inclusive design.

\section{References}

1. Aldersey-Williams, H., Bound, J., Coleman, R.: The methods lab: User research for design. Design for Ageing Network (1999). Avail. at www.education.edean.org (accessed Feb 09)

2. Bajekal, M., Harries, T., Breman, R., Woodfield, K.: Review of Disability Estimates and Definitions. Department for Work and Pensions, London (2004)

3. Clarkson, P.J., Coleman, R., Hosking, I., Waller, S.: Inclusive design toolkit. EDC, Cambridge, UK (2007). Avail. at www.inclusivedesigntoolkit.com (accessed Feb 09)

4. Department of Social Security Social Research Branch, Disability Follow-up to the 1996/97 Family Resources Survey [computer file]. Colchester, Essex: UK Data Archive [distributor], 3 March 2000. SN: 4090

5. Dong, H., Keates, S., Clarkson, P.J.: UK and US industrial perspectives on inclusive design. In Proceedings of Include, Helen Hamlyn Centre, London, UK, March 2003

6. i design project website. www-edc.eng.cam.ac.uk/idesign3/ (accessed Feb 09)

7. Persad, U., Langdon, P.M., Clarkson, P. J.: Characterising user capabilities to support inclusive design evaluation. Int. J. Universal Access Information Society 6(2):119-135. (2007)

8. The Philips Index: Calibrating the Convergence of Health care, Lifestyle and Technology. Available at www.philipsindex.ca (accessed Feb 09)

9. Poulson, D., Ashby, M., Richardson, S.: USERfit: A practical handbook on user-centred design for rehabilitation and assistive technology. HUSAT Research Institute for the European Commission. (1996)

10. Steinfeld, A., Steinfeld, E.: Universal design in automobile design. In: Preiser, W., Ostroff, E. (eds) Universal design handbook, McGraw-Hill, New York, pp 50.1-50.13 (2001)

11. Waller, S., Langdon, P.M., Clarkson, P.J.: Using disability data to estimate design exclusion. Int. J. Universal Access Information Society. In press. 